\title{
NOVAE IN CLUSTERS AND GALAXIES
}

\author{
Michael Shara \\ Space Telescope Science Institute \\ 3700 San Martin Drive \\ Baltimore, MD 21218
}

\section{Introduction}

My main goal in giving this review is NOT to induce hibernation in the audience. Rather, it is to survey the known cases of erupting classical novae in open and globular clusters, and galaxies external to the Milkey Way. More important, I want to emphasize what these novae in stellar systems can teach us about nova eruptions. In my conclusions I will make a few brief remarks about what we might hope to know about classical novae by the time IAU Symposium \#222 occurs (by then Space Telescope will hopefully have been in orbit and producing scientific results for many years!). References to older works may be found in the reviews of Trimble (1980) and Webbink (1980).

\section{Novae and Related Systems in Open Clusters}

Not a single classical nova is known to have occurred with absolute certainty in any open cluster. Kholopov (1956) has suggested that the nova V720 Sco which reached photographic magnitude 7.5 in 1950 , is a member in the galactic cluster NGC 6475 . Identification of the quiescent object is uncertain (Duerbeck 1987). The object may be fainter than 21 magnitude. Careful astrometric study of the original plate material and/or very deep $\mathrm{U}, \mathrm{B} C \mathrm{CD}$ images will be needed to recover the old nova. A radial velocity of the object (even more difficult) will be necessary to determine conclusively whether it really is a member of the open cluster. The age of NGC 6475 is $7 \times 10^{7}$ years. Thus determination of the cluster membership or not of V720 Sco is a valuable constraint on the evolutionary time necessary for a binary to reach the mass transfer rate necessary for nova eruptions.

Sion et al (1989) have detected a cool expanding shell about the binary V471 Tau in the Hyades open cluster. The large expansion velocity detected by these authors (components of $-575 \mathrm{~km} \mathrm{~s}^{-1}$ and $-1230 \mathrm{~km} \mathrm{~s}^{-1}$ ) strongly suggest the possibility that this shell is the remnant of an ancient nova outburst. Sion et al (1989) also note that a nova was recorded by Chinese observers in 396 A.D. within a degree on the sky of the location of V471 Tau. Further observations, particularly with the Hubble Space Telescope to try to resolve the ejected shell, are clearly warranted. With an age of only $5 \times 10^{8}$ years this possible old nova in the Hyades offers the opportunity for a second interesting constraint on the evolutionary time scale demanded of binaries before the nova phase is possible. 
Gilliland and Brown (1989) have recently discovered a catacylsmic binary in the very old open cluster M67. The 2.089 hour period and extremely blue color (U-B=-0.9) assure that the object is indeed a cataclysmic binary. What is extremely interesting about this object is the extraordinary faintness deduced from the apparent magnitude and known distance of the cluster; $M_{V}=11.3$. This is one of the intrinsically faintest cataclysmic binaries known. While there is no evidence that it has erupted as a nova, it is one of the only cataclysmics whose age we know accurately. The great age and faintness of this object, and what it can tell us of close binary evolution, make it worthy of very detailed study. are that

The main lessons to be drawn from the few cataclysmics known in open clusters

1. cataclysmics can, in fact be made in open clusters; and

2. they must be ejected as the clusters dissolve. This should lead to a flattened, old population I-like distribution for cataclysmics in the Milkey Way, and presumably a similar distribution in M31. The distribution of novae in M31 (Ciardullo, 1987) is distinctly population II. This may suggest a two population model of novae; some produced in open clusters in the spiral arms and others produced in the bulge and/or globular clusters.

\section{Novae in Globular Clusters.}

Only two Galactic classical novae are known with certainty to have erupted in globular clusters in the last one-hundred and forty years. The history of T Sco (Nova Sco $1860)$ has been reviewed in detail by Sawyer (1938). This moderately fast nova $\left(t_{3} \approx 24\right.$ days) was seen by several observers right at the core of the globular cluster M80.

Nova Oph 1938 has been tentatively identified by Shara et al (1986) as a faint, crowded, UV-bright star at the position specified by Hogg and Wehlau (1964). A noisy spectrum of the object (Shara, Moffat and Potter 1990) shows $\mathrm{H} \alpha$ in emission. This provides near-certain confirmation of the suggested candidate. The detailed study of the object will have to await the launch of the Hubble Space Telescope.

The lessons to be learned from these novae in globular clusters are that

1. Novae can erupt in very low $Z$ systems. This is supportive of the theoretical models which posit that the high $\mathrm{Z}$ abundances seen in nova ejecta are due to metals dredged up from the underlying white dwarf.

2. The maximum magnitude-rate of decline relation is the same for the nova in M80 as others of comparable speed in the galaxy and M31. This, too, is in accord with suggestions that nova light curves and the MMRD are the same at all times and places. (Shara, 1981). 


\section{Novae in Galaxies.}

Most of what we know about the eruption of classical novae comes from detailed photometric and spectrophotometric observations of classical novae in the Galaxy. There is much to be learned however, from the spatial distribution and eruption frequency of large samples of novae in different types of galaxies.

We can't see novae far enough away in our own galaxy to be able to accurately compare their distribution with that of the novae erupting in M31. We can, however, try to carry out the most accurate census of local neighborhood novae and related systems possible to determine accurately the space density of these objects. The paper by Shara, Moffat and Potter elsewhere in these proceedings describes a very deep survey for Galactic UV-bright objects. Eight cataclysmic and cataclysmic-like objects have been found in one square degree to a limiting magnitude $B \approx=20$. This implies a local space density of $\approx=10^{-4} p c^{-3}$ for cataclysmics. Hibernation theory predicts a similar space density.

Graham (1984) summarized the distribution of novae in the Large and Small Magellanic Clouds. Novae seem to strongly avoid the bars of both galaxies. Instead, they congregate outside the main bodies of each galaxy. A possible interpretation of this space distribution is that the novae in the LMC and SMC are all very old systems, but further study is warranted.

The advent of modern CCD detectors has enabled several dramatic advances in our knowledge of extragalactic novae. The multi-year survey of Ciardullo et al (1987) has finally revealed the true spatial distribution of novae in the central part of M31. To a distance of 12 arc minutes from the nucleus the novae follow the light of the bulge almost exactly.

Ciardullo et al (1987) were able to detect novae right up to the center of M31 by observing in narrow-band $\mathrm{H} \alpha$ filters. The apparent absence of novae in the inner 2 arc minutes of M31 (Arp 1956) was shown to be due to the inability of photographic plates to detect novae very close to the bright center of the galaxy. Ciardullo et al (1987) were also able to show that novae remain bright in $\mathrm{H} \alpha$ long after they had faded in $\mathrm{B}$. A six week run at the Wise Observatory, together with data from KPNO and the University of Texas has now quantified the relationship between the $\mathrm{H} \alpha$ and $\mathrm{B}$ light curves. (See Figure 1). The $\mathrm{H} \alpha$ light curves do, indeed, remain several magnitudes brighter than the $B$ light curves for many months after the eruption. Surprisingly, the rates of decline of the $B$ and $H \alpha$ light curves are, to within the observational error, exactly the same for the $\approx 10$ novae with good and extensive data. This strongly implies (but does not prove) that both the $\mathrm{B}$ continuum and $\mathrm{H} \alpha$ emission are produced in the shell ejected from a nova. These observations are extremely strong constraints on theoretical spectra produced by time dependent, radiative transfer computer codes. Any successful simulation of the time dependent spectral evolution of a nova shell must reproduce these light curves. 

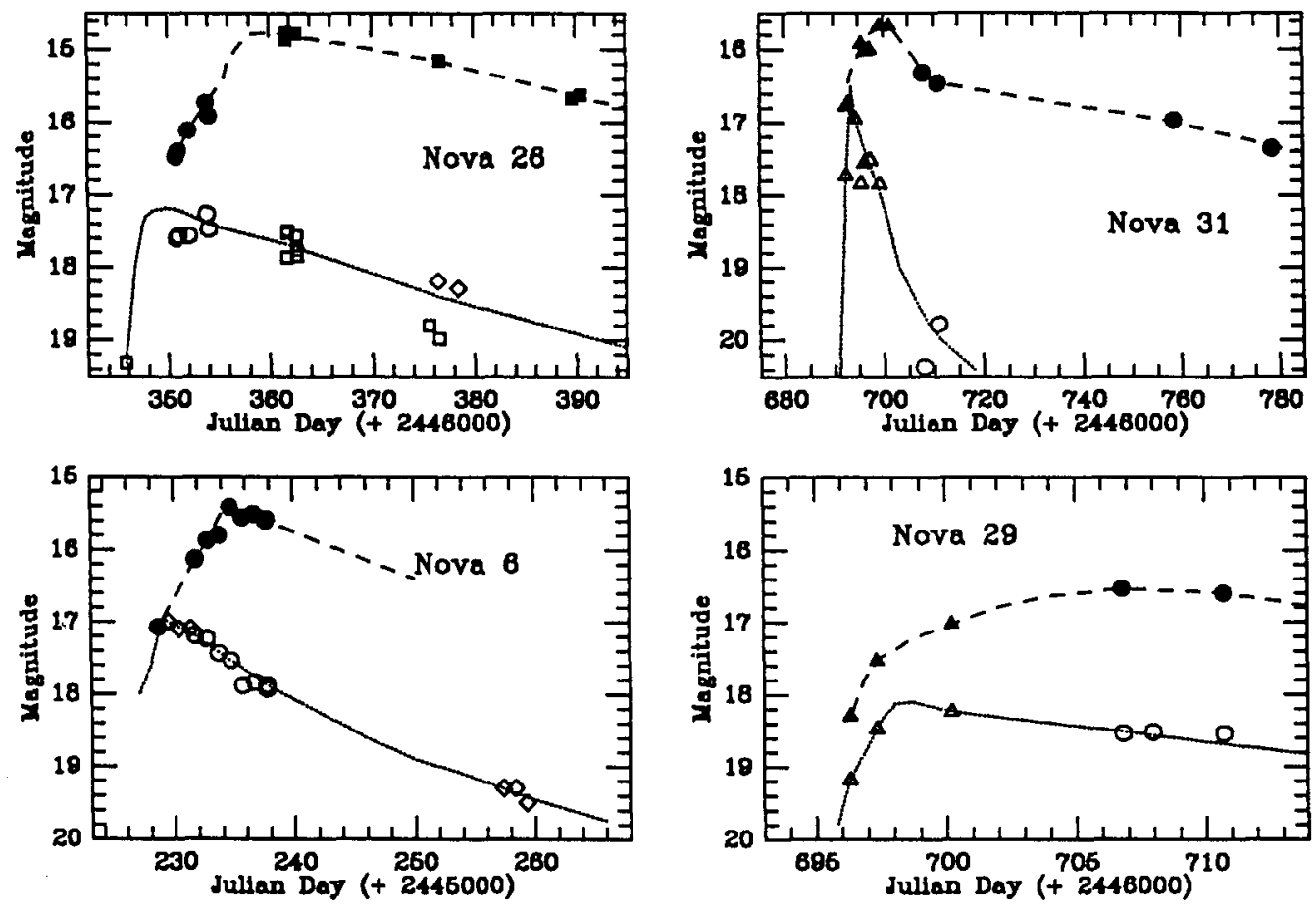

$\mathrm{H} \alpha$ emission is clearly a powerful way to locate novae in external systems, and several groups are using it to carry out surveys in other external galaxies. For example, Ford, Ciardullo and Williams are surveying NGC 5128 (Cen A) for erupting nova with the CTIO 4 meter. Both this survey, and the ongoing CCD surveys of M31 by Capaccioli et al will be important to check the apparently bimodal distribution of the brightness of the novae at maximum magnitude in a given galaxy. Also important to check will be whether extremely bright novae (similar to V1500 Cyg 1975, CP Puppis 1942) occur frequently in other galaxies. Several speakers have already referred to such bright novae, and I want to repeat their suspicion that we may be seeing the most massive white dwarfs, and/or strongly magnetic systems, and/or oxygen-neon-magnesium white dwarfs in these very bright novae.

The distance record for nova detection must certainly rest with Pritchet and van den Bergh (1987). They have succeeded in detecting nine novae in the Virgo Cluster by taking advantage of the exquisite seeing at the Canada-France-Hawaii Telescope. Though their light curves were sparsely sampled, they were able to determine a distance of $\simeq 20$ Megaparsec to the cluster, and a Hubble constant $\mathrm{H}_{0} \approx=69 \pm 14 \mathrm{~km} \mathrm{~s}^{-1} \mathrm{Mpc}^{-1}$. These observations strongly suggest that novae should be detectable in the Coma Cluster with the Hubble Space Telescope. 


\section{The Hubble Space Telescope.}

I want to close by noting that the "Nova Community" may be an important beneficiary of the Hubble Space Telescope program. We will certainly be seeing ultra-high resolution pictures of nova shells, and spectra of cataclysmics in globular clusters during the first year of HST operations, because these are Guaranted Time Observers programs. High resolution, ultraviolet spectra, both of Galactic and M31 novae should start becoming available a few years after launch. Ultra-high time resolution photometry is also possible ( 14 microsecond sampling can be achieved with the high speed photometer) both in visible and UV light. The distances of a few very closeby cataclysmics might be directly measurable with the astrometric instrumentation on HST, while distances as far away as the Coma Cluster may also be determinable from the MMRD relation if novae can be detected in those very distant galaxies. The successful launch and operation of HST will surely present many important new facets of nova behavior for our study by the time of IAU Colloquium \#222.

\section{References}

Arp, H. C. (1956), A.J. 61, 15.

Ciardullo, R., Ford, H. C., Neill, J. D., Jacoby, G. H. and Shafter, A. W. (1987), Ap.J. $318,520$.

Ciardullo, R., Shafter, A. W., Ford, H. C., Neill, J. D., Shara, M. M. and Tomaney, A. B. (1990), Ap.J. submitted.

Duerbeck, H. W. (1987), Space Science Reviews 45, 1.

Gilliland, R. and Brown, T. (1989), private communication.

Hogg, H. B. and Wehlau, A. (1964), J.R.A.S. Canada, 58, 163.

Kholopid, P. N. (1956), P.Zu. 11, 325.

Pritchet, C. J. and van den Bergh, S. (1987), Ap.J. 318, 507.

Sawyer, H. B. (1938), J.R.A.S. Canada 32, 69.

Shara, M. M. (1981), Ap.J. 243, 926.

Shara, M. M., Moffat, A. F. J., Potter M., Hogg, H. S. and Wehlau, A. (1986), Ap.J. 311, 780.

Sion, E., Bruhweiler, F. C., Mullon, D. and Carpenter, K. (1989), Ap.J. 341, 47.

Trimble, V. (1980), in "Star Clusters", J. E. Hesser, ed., IAU Symposium 85, D. Reidel, Dordrecht, p. 259.

Webbink, R. F. (1980), in "Close Binary Stars: Observations and Interpretation", Plavec, M. J., Popper, D. M. and Ulrich, R. K. eds., IAU Symposium 88, D. Reidel, Dordrecht. 